Journal of

Dentistry and Oral Health

\title{
Dosimetrical Relationship between 3d Delineation and Oral Complications of Radiotherapy in Patients with Head and Neck Cancer
}

\section{Raylane Farias de Albuquerque ${ }^{1}$, Luiz André Nadler Lins ${ }^{2}$, Igor Henrique Morais Silva ${ }^{3 *}$}

${ }^{1}$ Department of Oral Oncology, Hospital de Câncer de Pernambuco (HCP - PE), Recife, Pernambuco, Brazil

${ }^{2}$ Department of Radiotherapy, Instituto de Radioterapia Waldemir Miranda (IRWAM), Recife, Pernambuco, Brazil

${ }^{3}$ Department of Oral Oncology, Hospital do Câncer de Pernambuco (HCP - PE), Recife, Pernambuco, Brazil

${ }^{\star}$ Corresponding author: Igor Henrique Morais Silva, Department of Oral Oncology (Dentistry) - Hospital de Câncer de Pernambuco,Avenida Cruz Cabugá 1597 - Santo Amaro, Recif-PE. Brasil, CEP 50040-000,E-mail: igorrecife@hotmail.com

Received Date: March 07, 2018; Accepted Date: May 02, 2018; Published Date: May 05, 2018

Citation: Raylane Farias de Albuquerque (2018) Dosimetrical Relationship between 3d Delineation and Oral Complications of Radiotherapy in Patients with Head and Neck Cancer. J Dent Oral Health 5: 1-8.

\section{Abstract \\ Purpose: To study the relationship between the dosimetric parameters of the radiotherapy contouring protocols for head and neck cancers and the acute oral complications resulting from this treatment. \\ Methods: This was a cross-sectional, quantitative study. Radiotherapy was planned according to established design protocols. The constraints used were those described in QUANTEC (Quantitative Analyses of Normal Tissue Effects in the Clinic) and the complications were classified based on the CTCAE “” (National Cancer Institute's Common Criteria Chart of Toxicity). \\ Results: Eleven patients participated, most of them male (89\%), with a mean age of 60.2 years. The most frequent tumor site was oropharynx (55\%) and the degree of staging IV (64\%). Among the participants, $73 \%$ were smokers and $82 \%$ had a habit of drinking alcohol. The most frequent oral complications were dysphagia (91\%), xerostomia (82\%), mucositis (54\%) and oral pain (54\%). For the complications dysphagia, xerostomia and mucositis, relationships were found between the dosimet- ric data and the development of these, for oral pain the difference between the groups was not significant. \\ Conclusion: Dosimetric data are directly related to the development of certain oral complications resulting from radio- therapy treatment for malignant head and neck neoplasms.}

Keywords: Head and neck neoplasms; Radiotherapy; Organ at risk; dose volume histogram; oral oncology; oral oncology 


\section{Introduction}

The estimated incidence of cancer in Brazil for the year 2018 indicates the occurrence of approximately 14,700 new cases of neoplasms of oral cavity and 7,670 new cases of larynx. Especially in the Northeast region of Brazil is estimated 2,810 new cases of oral cavity cancer, for both sexes [1].

Radiotherapy and Surgery are described as the standard therapies for initial and locally advanced malignant tumors in the head and neck region [2,3]. For initial lesions it is recommended to perform local surgery with or without neck dissection. For more advanced stages, radiotherapy, associated or not with chemotherapy, is recommended as a complement to the surgical treatment or can be used as a radical or palliative treatment, also associated or not with chemotherapy $[4,5]$.

In radiation therapy, an energy charge is directed to eliminate malignant cells in a specific area. Radiation causes DNA damage leading to the death of normal and neoplastic cells [6]. The major challenge of radiotherapy treatment is to maximize disease control, minimizing morbidity and toxicity in surrounding normal tissues [7]. Radiotherapy is typically associated with acute and late toxicity that can have profound effects on the patient's quality of life. Common acute toxicities of head-neck irradiation include mucositis, dermatitis, dysphagia, and odynophagia. These complications may lead to prolongation or interruption of treatment with potential adverse impact on results $[7,8]$.

Several radiotherapy techniques can be used to treat head and neck cancer. Conventional radiotherapy, also known as $2 \mathrm{D}$ Radiotherapy (RT2D), is performed by delimiting the volume of treatment from plain radiographs. Conformational radiotherapy, also known as $3 \mathrm{D}$ radiation therapy (RT3D), has been developed so that the planning is performed from computerized tomography imaging with the visualization of the tumor or target and the normal tissues in volumetric form. The images are exported to software that allows planning the delivery of doses through different input portals known as treatment fields [6]. With current planning software, the relationship between radiation doses and tumor volumes and adjacent normal tissues is expressed as dose-volume histograms [9].ing to smoker people the studies shows that's smoking is more prevalent in depression patients [23,24]. And among the many harmful oral habits, which are believed to be induced by emotional disturbances, smoking is possibly the most important in relation to worsened periodontal conditions [25].

The use of dose-volume histograms for treatment plan analysis has become indispensable for dose analysis in the tumor and in normal tissues. Thus, with the development of the three-dimensional technique, a greater individualization of treatments was possible. Radiation doses are better shaped and, compared to the conventional technique, safety has been increased with better visualization and evaluation of treated anatomical structures [10]. Dosimetric data on tolerance of normal tissues began to be described in the literature retrospectively from these computational data of radiotherapy planning [11].
The improvement of the radiotherapy technique, with the presence of more targeted doses and protection of the risk organs, has allowed the reduction of toxicities, with mucositis being the most frequent acute toxicity and xerostomia the most observed late toxicity. The introduction of threedimensional radiotherapy (3D-CRT) and intensity-modulated radiotherapy (IMRT) systems allows greater protection of the major salivary glands preserving the salivary flow rate. With this, patients' quality of life was improved, as well as the risk of damage to the teeth induced by radiation [12]. Knowing that the oral complications resulting from radiotherapy treatment directly influence the patient's quality of life and adherence to treatment, the present study aimed to evaluate the dosimetric relationship between the three-dimensional design and the acute oral complications of radiotherapy in patients withcancer of the head and neck diagnosed in the Hospital de Câncer de Pernambuco.It is a pilot study due to the unprecedented characteristic of the association between the radiotherapy design and the oral complications.

\section{Methods}

The study was a prospective, quantitative approach. The study population consisted of 11 oncology patients from a Reference Hospital in Oncology in the state of Pernambuco, who underwent $3 \mathrm{D}$ conformational radiotherapy in the head and neck region and were followed up at the Odontology clinic of this hospital.Patients older than 18 years, with diagnosis of Epidermoid Carcinoma in the head and neck region (oral cavity, oropharynx, hypopharynx, larynx and nasopharynx), whose therapeutic indication involved exclusive conformational radiotherapy, concomitant with chemotherapy or as adjuvant to surgery were included in the study. Those with performance status below 70 according to Karnofsky score, diagnosis of head and neck cancer of other locations (skin lesions or metastases), cognitive deficit or refusal of the Free and Informed Consent Term, were excluded.The planning of radiotherapy was performed according to the design protocols established in previous studies [13-16]; the constraints used were those described in the set of articles known as Quantitative Analyzes of Normal Tissue Effects in the Clinic (QUANTEC) [17], the evaluation of dosimetric parameters was performed through dose-volume histograms and described in its own form.After signing the Informed Consent Term, the patients were examined weekly throughout the period of radiotherapy,at the Department of Dentistry in order to identify the presence of oral complications of radiotherapy. It used its own form, filled out according to clinical examination. To determine and classify complications, the National Cancer Institute's Common Criteria Chart of Toxicity (CTCAE) v4.03 $[18,19]$ was used as a reference. Oral complications included angular cheilitis, dental caries, dry mouth (xerostomia), dysphagia, gingival pain, mucositis, oral fistula, oral bleeding, oral pain, periodontal disease, toothache, radiodermatitis and osteoradionecrosis. Complications were recorded on a form according to the degree presented, on a weekly basis, during the period of radiotherapy (thirty-five sessions or seven weeks). 
The follow up and dental treatment that the patients participating in the research received, in no way differ, from what is already done in a standard way for all head and neck patients who undergo radiotherapy treatment. Once the patients were evaluated and appropriate pre-radiotherapy, they were followed up throughout the treatment period, according to protocols adopted in the Department of Dentistry of the Hospital de Câncer de Pernambuco.Data were analyzed through a descriptive analysis of patient data and complications and their respective frequencies. Subsequently, the complications and the dosimetric data of patients with or without complications were analyzed. The mean values of the dosimetric data of the volumes of structures relevant to each type of complication that received certain percentages of doses (V\%) were compared in groups that had or had no complications through radar-type graphics. Excel software was used for the analyzes. The work was approved by the institution's Ethics and Research Committee E.

\section{Results}

Eleven patients participated, most of them male (89\%), with a mean age of 60.2 years, minimum age of 38 and maximum of 75 years. Stratified in age groups, $27 \%$ were less than 50 years old, $27 \%$ were between 50 and 64 years old and $46 \%$ were elderly. Most of the patients were retired (36\%) and the rest were divided between bricklayer (27\%), self-employed (18\%), carpenters (9\%) and realtors (9\%) (Table 1).

\begin{tabular}{|ll|}
\hline Sociodemographic characteristics & \multicolumn{1}{c|}{ Numbers (\%) } \\
\hline Age (inyears)' & $60,2 \pm 12,7(38 ; 75)$ \\
\hline Age group & $3(27,0 \%)$ \\
\hline$<50$ yearsold & $3(27,0 \%)$ \\
\hline $50-64$ years old & $5(46,0 \%)$ \\
\hline$>$ or $\bullet 65$ years old & \\
\hline Sex & $2(18,0 \%)$ \\
\hline Female & $9(89,0 \%)$ \\
\hline Male & \\
\hline Profession & $4(36,0 \%)$ \\
\hline Retired/Pensioner & $2(18,0 \%)$ \\
\hline Autonomous & $1(9,0 \%)$ \\
\hline Woodworker & $1(9,0 \%)$ \\
\hline Realtor & $3(27,0 \%)$ \\
\hline Bricklayer & Minimn) \\
\hline
\end{tabular}

-Mun = standarddl''iatioo (Ma.ximwn ;Minimwn)

Table 1: Sociodemographic profile of patients diagnosed with malignant lesions in the head and neck region attended at the Hospital de Câncer de Pernambuco.

Regarding alcohol or smoking habits, $73 \%$ reported being smokers or former smokers, and $82 \%$ reported frequent alcohol consumption (Table 2). Regarding alcohol or smoking habits, $73 \%$ reported being smokers or former smokers, and $82 \%$ reported frequent alcohol consumption (Table 2).

\begin{tabular}{|ll|}
\hline Variables & Numbers $(\%)$ \\
\hline Smoking & \\
\hline Yes & $8(73,0 \%)$ \\
\hline No & $3(27,0 \%)$ \\
\hline Alcoholism & \\
\hline Yes & $9(82,0 \%)$ \\
\hline No & $2(18,0 \%)$ \\
\hline
\end{tabular}

Table 2. Life habits of patients with diagnosis of malignant lesions in the head and neck region treated at the Hospital de Câncer de Pernambuco.

As to the clinical aspects of the patients, all had histology of Epidermoid Carcinoma, most of the tumors were located in the oropharynx region (55\%), followedby $18 \%$ in the oral cavity, $18 \%$ in the larynx and $9 \%$ in the nasopharynx. According to the degree of staging of the tumor, $64 \%$ of the patients had grade IV, $18 \%$ grade III and $18 \%$ grade II. Of the 11 patients investigated, 73\% underwent chemotherapy and 36\% underwent surgery prior to radiotherapy (Table 3).

\begin{tabular}{|ll|}
\hline Clinical characteristics & Numbers (\%) \\
\hline Tumor diagnosis & $11(100 \%)$ \\
\hline Squamous Cell Carcinoma & $6(55,0 \%)$ \\
\hline Tumor Location & $2(18,0 \%)$ \\
\hline Oropharynx & $2(9,0 \%)$ \\
\hline Larynx & I $(9,0 \%)$ \\
\hline Oral cavity & \\
\hline Rhinopharynx & $0(0,0 \%)$ \\
\hline Degree of staging & $2(18,0 \%)$ \\
\hline I & $2(18,0 \%)$ \\
\hline II & $7(64,0 \%)$ \\
\hline m & $8(73,0 \%)$ \\
\hline IV & $3(27,0 \%)$ \\
\hline Chemotherapy & $4(36,0 \%)$ \\
\hline Yes & $7(64,0 \%)$ \\
\hline No & \\
\hline Surgery & \\
\hline Yes & \\
\hline No & \\
\hline
\end{tabular}

Table 3. Clinical aspects of patients diagnosed with malignant lesions in the head and neck region treated at the Hospital de Câncer de Pernambuco.

Among the complications evaluated in the patients, dysphagia, present in about $90 \%$ of patients, followed by dry mouth (81\%), mucositis (54\%) and oral pain (54\%). Among the least incidents are radiodermatitis (18\%), oral bleeding (9\%) and cheilitis (9\%). Complications Dental caries, gingival pain, oral fistula, periodontal disease, tooth pain and osteoradionecrosis were not present (Figure. 1). 


\section{Incidence of Complications}

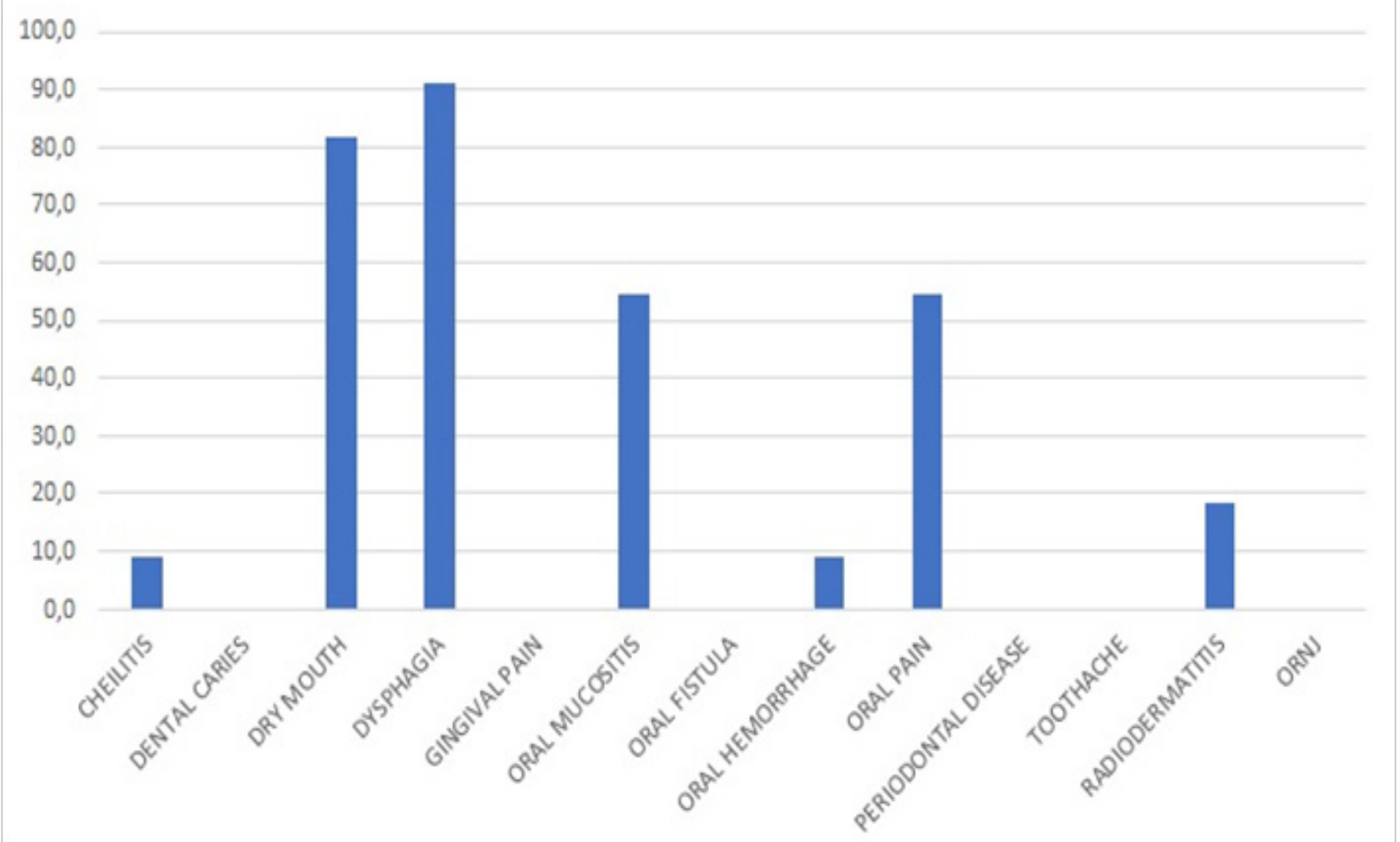

Figure 1. Incidence of complications assessed in patients with head and neck neoplasms submitted to conformational radiotherapy (3D), during treatment.

When analyzing the mean dosimetric data of patients who had dysphagia or not; it is observed that in relation to the oral cavity, all V\% were higher in the group that presented some degree of dysphagia. Regarding the doses in the pharyngeal contrictor muscles, this relation was not observed. This can be partially explained by the high average doses received in both groups (with and without dysphagia) (Figures. 2a and 2b).

\section{V\% in the constrictors of the pharynx $x$ Dysphagia}

\section{V\% in the oral cavity $x$ Dysphagia}

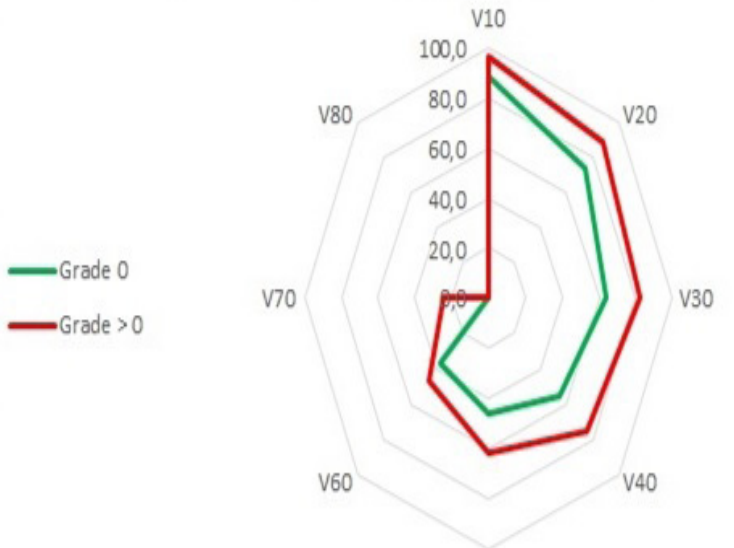

V50
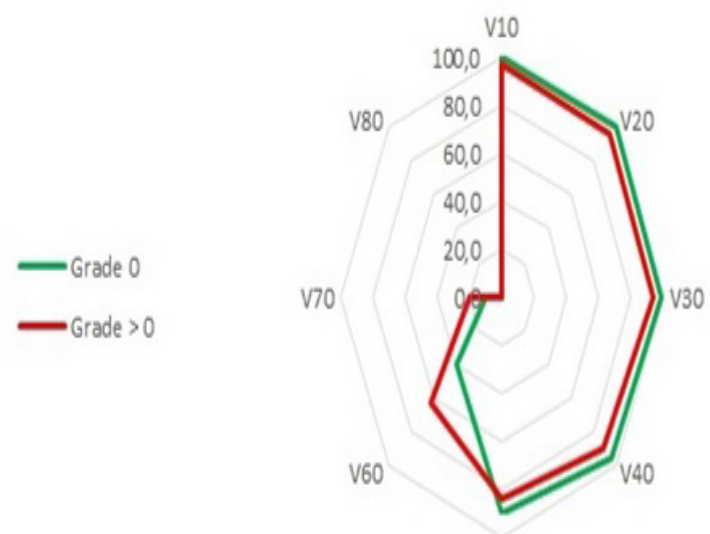

V50

Figures $2 \mathrm{a}$ and $2 \mathrm{~b}$. Relationship between the volumes of oral cavity and constrictor muscles of the pharynx irradiated and the development of dysphagia. 
In relation to dry mouth, we found that patients with lower degrees ( 0 and 1 ) had higher mean doses in the parotid and submandibular muscles, compared to patients who presented higher degrees of dry mouth $(>1)$. This antagonistic result can be explained by the fact that the mean doses were high in both groups, therefore, the comparison should be made taking into consideration the minimum doses. Then, in the group that presented lower degrees of dry mouth ( 0 and 1$)$, the minimum dose was lower than the group with dry mouth grade> 1 , that is, a larger volume of the parotid was preserved in the group that presented lower dry mouth (Figures. $3 \mathrm{a}$ and $3 \mathrm{~b}$ ).

\section{V\% in the parotidx Dry Mouth}

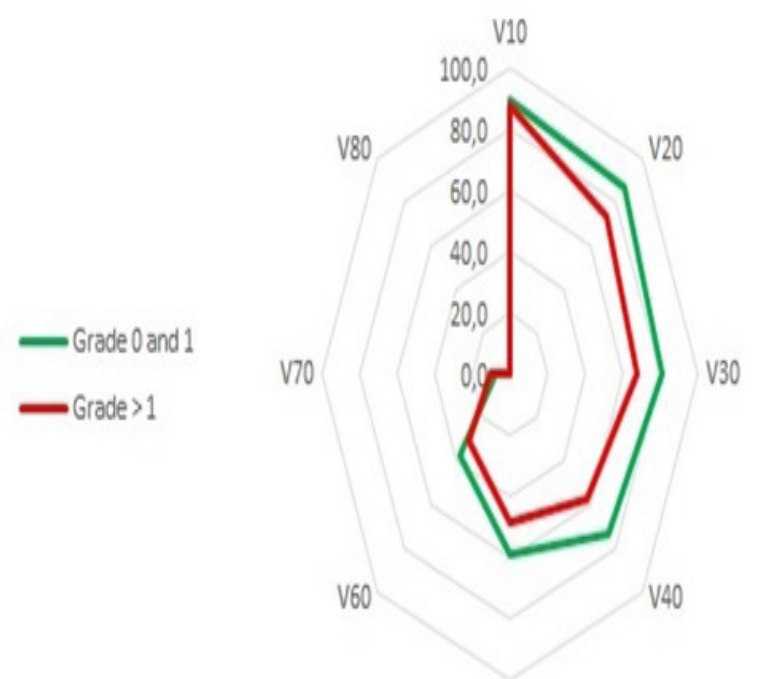

V50

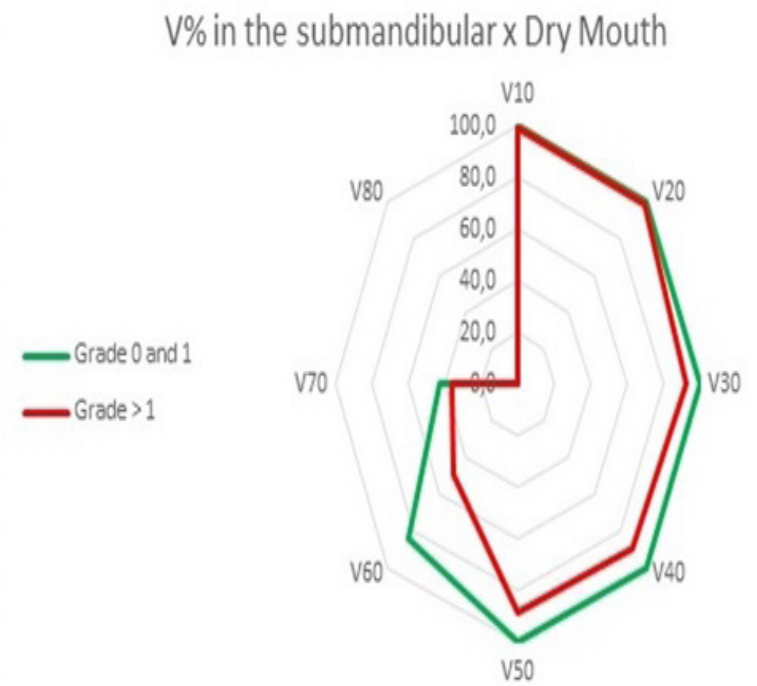

Figures $3 \mathrm{a}$ and $3 \mathrm{~b}$. Relationship between the volumes of parotid glands and submandibular glands irradiated and the development of dry mouth.
For mucositis, differences were observed in oral cavity and buccal mucosa doses, among groups that developed or not. For oral cavity, it was observed that V10, V20, V30, V40 and V70 were higher in the group that developed the complication (mucositis), that is, in these established parameters, a larger percentage of the structure received a higher dose or equal to 10, 20, 30, 40 or 70 Gy respectively. For V50, V60 and V80 no significant differences were observed. For the buccal mucosa all V\% were higher in the group that developed mucositis (Figures. 4a and 4b).

\section{V\% in the oral cavity x Mucositis}

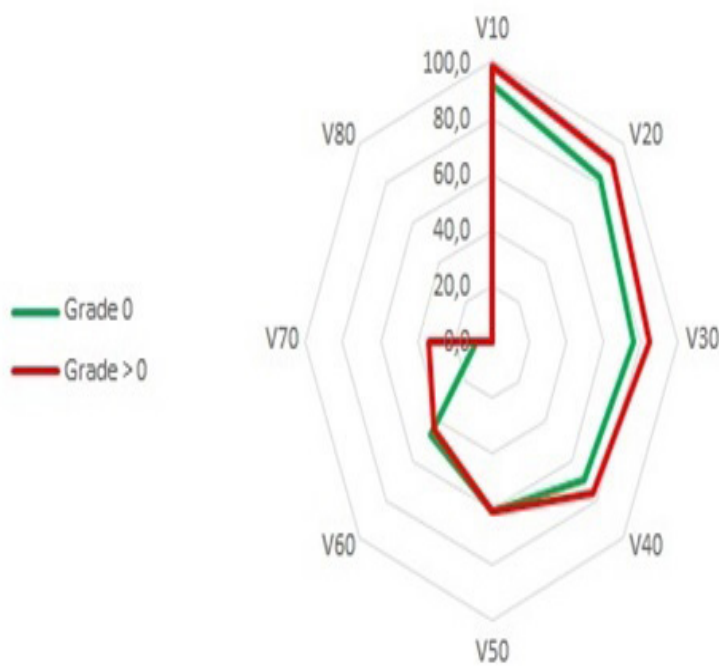

\section{V\% in the buccal mucosa x Mucositis}

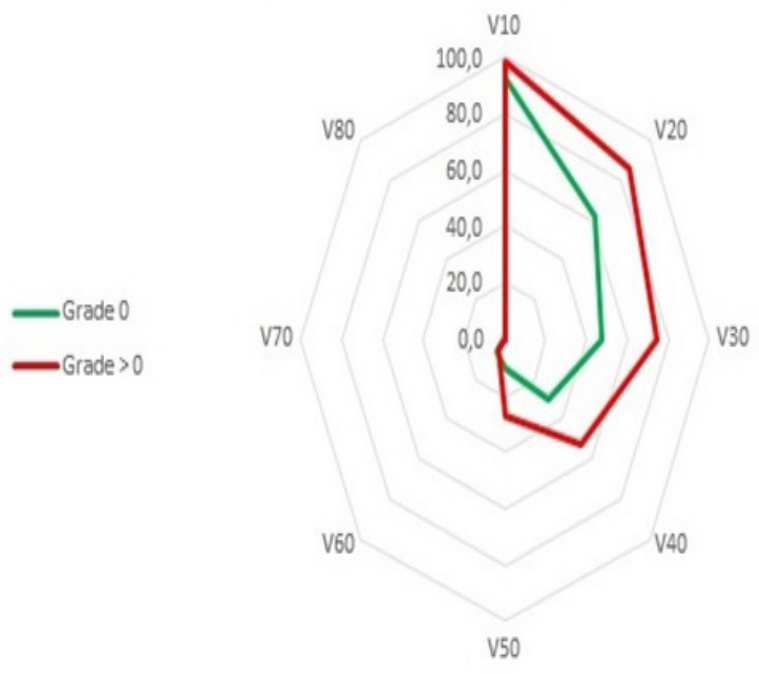

Figures $4 \mathrm{a}$ and $4 \mathrm{~b}$. Irradiated oral cavity and buccal mucosa volumes and the development of mucositis. 
We did not find significant dosimetric differences between the volume of oral cavity irradiated and the development of oral pain in the sample analyzed in this study (Figure. 5).

\section{V\% in the oral cavity $x$ oral pain}

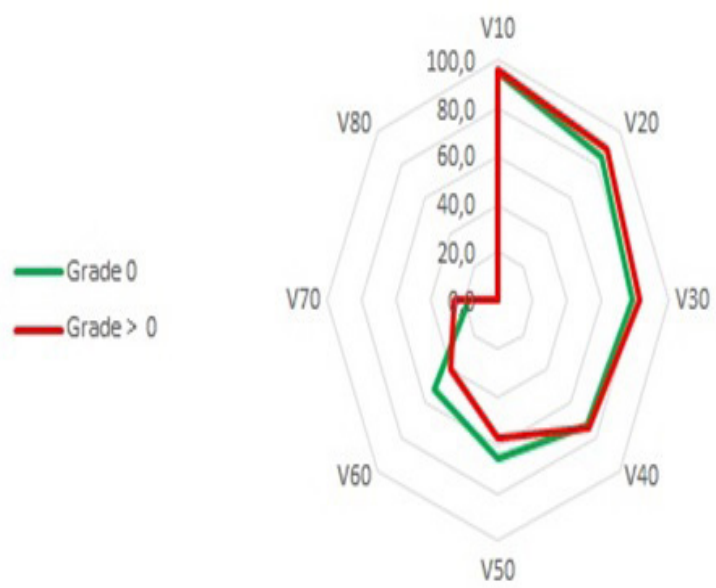

Figure 5. Relationship between the volume of oral cavity irradiated and the development of oral pain.

One of the patients in the group did not present any degree of complication. When comparing the mean dose (Dmean) in the several analyzed structures, with the means of the Dmean of the other patients; it was observed that in the patient that did not develop complications the Dmean were smaller in the mandible, parotid, oral cavity, lips, buccal mucosa and in the submandibular and greater in the maxilla, constrictors of the pharynx and in the larynx (Figure. 6).

\section{Mean dose $x$ Complications}

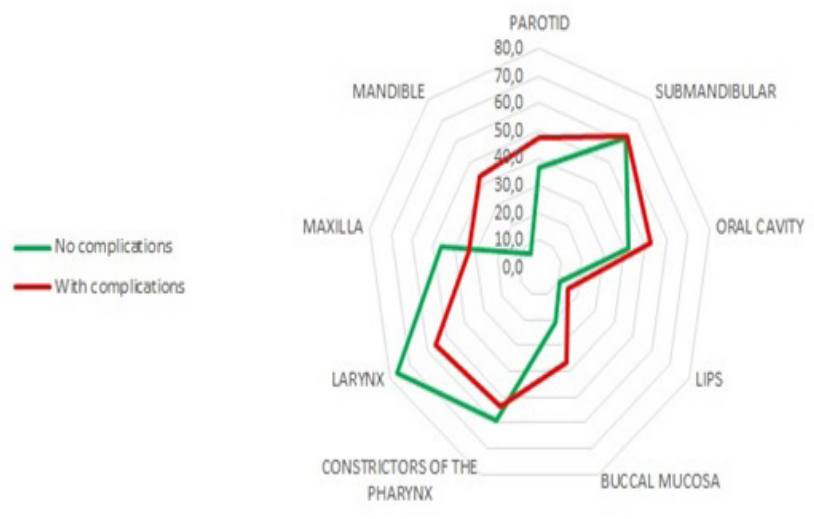

Figure 6. Relationship between the mean dose (Gy) received by each structure and the development of complications.

\section{Discussion}

The cases of cancer diagnosed on the head and neck region represent fifth in the list of the most frequent neoplasms $[20,21]$. The most frequent histological type is squamous cell carcinoma, accounting for about $90 \%$ of lesions of the oral cavity and larynx. The most important risk factor is smoking, in synergy with alcoholism. Other factors are important such as HPV infections, vitamin deficiency and immunosuppression [22]. The histological type of squamous cell carcinoma was selected as inclusion criterion since work, considering its greater frequency in head and neck neoplasms. The most common location was oropharynx. Among the participants in the study, $73 \%$ had a history of chronic smoking and $82 \%$ of alcoholism, reinforcing the already established relationship of habits with the development of neoplasms in the head and neck region.

The findings of most studies on the prevalence of squamous cell carcinoma in males corroborate with those found in this study, where the majority of the sample consisted of men. National Cancer Institute (INCA) estimates for 2018 bring oral cavity tumors to 5th place among the 10 most common cancers for men, estimating for the same 11,200 new cases and 3,500 for women [1]. Data from the American Cancer Society (ACS) estimate for new cases in 2018 the 8 th position for men, with 37,160 cases. In contrast, both the Inca and ACS estimates do not bring the oral cavity and pharynx localization among the 10 most incident for women [23].

Radiotherapy and Surgery are described as the standard therapies for initial and locally advanced malignant tumors in the head and neck region [2,3]. Low stage injuries are usually treated with local surgery. In the more advanced stages, radiotherapy, associated or not with chemotherapy, is recommended as a complement to the surgical treatment $[3,4]$. Regarding the degree of staging, most of our sample presented stage IV, being to the therapeutic modalities according to the indicated in the literature, since more than two thirds of the patients were submitted to the chemotherapy and a small portion submitted to the previous surgery.

Complications arising from radiotherapy can be divided into acute and late complications. Because it is a restricted follow-up to the radiotherapy period, we evaluated the acute complications, finding dysphagia more frequent, followed by dry mouth, mucositis and oral pain. Up to $59 \%$ of the patients undergoing radiotherapy alone developed mild dysphagia and $25 \%$ severe dysphagia, this number rises to $69 \%$ in patients who received concomitant radiation and chemotherapy [24,25].

A factor that can contribute to the development of dysphagia is the presence of mucositis, a complication present in about $90 \%$ of patients receiving concomitant radiotherapy and chemotherapy, mucositis was found in more than half of our sample. Currently the association of chemotherapy has allowed a better local control of the disease and survival in these patients, in contrast has added an increase in the incidence of oral mucositis (MO). We can confirm this, since among the patients who did not have concomitant chemotherapy, the mucositis was non-existent or at most grade 1, with mild discomfort without diet modification. 
Despite the presence of mucositis in about $54 \%$ of the studied population, no patient presented severe degrees throughout the treatment period. Some of this success can be attributed to the protocol for the prevention of mucositis, with the use of the low-power laser to which the patients were submitted according to routine of the Department of Dentistry in the Hospital de Câncer de Pernambuco. The use of the laser in its preventive form presents effective results, delaying the onset of inflammation, decreasing the severity of the degrees and consequently the pain associated with $\mathrm{OM}$, as well as preventing complications with the use of a nasogastric tube and hospital admission [26-28]. The first clinical sign of mucositis is erythema appearing at cumulative doses of radiation to the head and neck of about $10 \mathrm{~Gy}$. Cumulating doses of $30 \mathrm{~Gy}$ (usually after two weeks) develop ulcers [29].

With this work we can observe that mucositis is especially related to the amount of dose received by the buccal mucosa, since all V\% were higher in the group that developed mucositis. For oral cavity, a significant difference was observed in V10, V20, V30, V40 and V70, which were higher in the group that developed the complication (mucositis). For V50, V60 and V80, no significant differences were observed between the two groups. As a comparison, the group that developed mucositis presented in the V30 about $85 \%$ of the volume of oral cavity receiving $30 \mathrm{~Gy}$ or more (cumulative dose in which the ulcers usually present), whereas the group that did not develop presented $76 \%$ of the structure receiving thesame dose.

Xerostomia or subjective sensation of dry mouth was present in about $81 \%$ of our sample. Radio-induced xerostomia depends on the cumulative doses of radiation on the head and neck region [30]. Doses of 20 Gy may cause a decrease in salivary flow, doses above 50 Gy cause severe glandular dysfunction, and a rapid decline in salivary flow may be observed during the first weeks of treatment [31]. It can be observed in the sample studied that patients who presented dry mouth grade $>1$ received in the parotid the average dose of approximately $41.6 \mathrm{~Gy}$, when the constraints indicate that the maximum dose allowed in the parotid is approximately $27 \mathrm{~Gy}$.

It can be observed that patients with lower degrees (0 and 1) had higher mean doses in the parotid and submandibular groups, compared to patients with higher degrees of dry mouth $(>1)$. This antagonistic result can be explained by the fact that the mean doses were high in both groups, therefore, the comparison should be made taking into consideration the minimum doses. In the group that presented lower degrees of dry mouth $(0$ and 1$)$ the minimum dose was lower in relation to the group with dry mouth grade $>1$, that is, a larger volume of the parotid was preserved in the group that presented lower degree of dry mouth. When possible, dose reduction for parotids leads to preservation of salivary function following radiotherapy. Xerostomia is a debilitating factor that can induce difficulty in swallowing, speaking and even facilitating the development of cavities.
When evaluating the data for oral pain, it is curious to observe that patients who presented grade $>0$ received the mean dose of $51.1 \mathrm{~Gy}$, while for grade $<0$ the mean dose was discreetly higher $51.6 \mathrm{~Gy}$, however, in patients who developed The volume of irradiated structure was larger, receiving larger doses up to V40. Oral pain is a subjective aspect of being evaluated, since such a symptom may be related to the development of oral mucositis, or even tumor pain itself.

In general, we can observe that patients who did not present complications received lower mean doses in the related risk organs compared to those who presented complications, which leads us to understand that the development of complications is directly related to the amount of dose received by each structure, as well as the volume of the organ receiving such dose. Recent studies have shown that intensity modulated radiotherapy (IMRT) is a technique that allows the administration of lower doses of radiation to normal tissue, maintaining or increasing the dose in the tumor, compared to the less advanced radiotherapy techniques.

With the knowledge that one of the major challenges with radiotherapy in head and neck cancer is to optimize disease control, decreasing toxicity in surrounding normal tissues and consequently morbidity for the patient, efforts over the past decades have bent over the techniques of planning and delivery of doses of radiotherapy, leading to improved dose distributions. These dosimetry gains, mainly the reduction of unnecessary irradiation of important adjacent structures, have resulted in gains in quality of life for the patient.

\section{Conclusion}

In view of the presented work, there is a relationship between the dosimetric parameters used in the three-dimensional design for the treatment of malignant neoplasms in the head and neck region and the oral complications resulting from this treatment. There is a need for further studies in the area in order to obtain more comprehensive evidence regarding the relationship between dosimetry and oral complications, aiming to improve patients' quality of life. 


\section{References}

1) (2018) Instituto Nacional de Câncer José Alencar Gomes Da Silva (INCA) - Prevention and Surveillance Coordination. Estimate 2018: incidence of cancer in Brazil. Rio de Janeiro: INCA.

2) Mour J, Lima R, Salvajoli J V, Souhami L, Faria S L (2013) Tumors of head and neck. In: (Org.). Radiation therapy in oncology. São Paulo: EditoraAtheneu . 505-515.

3) Lanzós i. et al. (2015) A critical assessment of oral care protocols for patients under radiation therapy in the regional University Hospital Network of Madrid (Spain). J Clin Exp Dent 7:. 613- 621.

4) Ang K (2011) A randomized phase III trial (RTOG 0522) of concurrent accelerated radiation plus cisplatin with or without cetuximab for stage III-IV head and neck squamous cell carcinomas (HNC). J ClinOncol 29: 5500.

5) Forastiere A et al. (2003) Concurrent chemotherapy and radiotherapy for organ preservation in advanced laryngeal cancer. N Engl J Med 349: 2091-2098.

6) Hara W (2015) General principles of radiation therapy for head and neck cancer. UpToDate.

7) Trotti A et al. (2003) Mucositis incidence, severity and associated outcomes in patients with head and neck cancer receiving radiotherapy with or without chemotherapy: a systematic literature review. Radiother Oncol 66: 253-62.

8) Langendijk J et al. (2008) Impact of late treatment-related toxicity on quality of life among patients with head and neck cancer treated with radiotherapy. J ClinOncol 26: 3770-3776.

9) Justino P, Carvalho H, Ferauche D, Ros Rs (2003) Planejamento tridimensional para radioterapia de tumores de esôfago: comparação de técnicas de tratamento e análise de probabilidade de complicações. RadiolBras $36: 157-162$.

10) Santos M, Garcia P (2013) The financial impact of the incorporation of IMRT and RapidArcTM techniques on shielding calculation of a linear accelerator. RevistaBrasileira de FísicaMédica. 7 : 61-64.

11) Wang X, Eisbruch A (2016) IMRT for head and neck cancer: reducing xerostomia and dysphagia. Journal of Radiation Research. 57 : i69-i75

12) Kuhnt T,Stang A ,Wienke A, Vordermark D, Schweyen R et al. (2016) Potential risk factors for jaw osteoradionecrosis after radiotherapy for head and neck cancer. RadiatOncol. 11: 101.

13) Freedman L (2015) A radiation oncologist's guide to contouring the larynx. PractRadiatoncol. 6: 129-30. Treatment of oral mucositis induced by radio and chemo-induced: comparison between conventional drug protocol and low intensity laser treatments. Dissertation. São Paulo: University of São Paulo; Institute of Energy and Nuclear Research, 2011.

14) Rouers $M$ et al. (2015) Maxillary and mandible contouring in patients with a head and neck area irradiation. PractRadiatOncol. 6: e61-72

15) Freedman L, Sidani C (2016) A Radiation Oncologist's Guide to Contouring the Parotid Gland. PractRadiatOncol.

16) Brouwer $C$ et al. (2015) CT-based delineation of organs at risk in the head and neck region: DAHANCA, EORTC, GORTEC, HKNPCSG, NCIC CTG, NCRI, NRG Oncology and TROG consensus guidelines. Radiotherapy Oncol J European SocTher Radiology Oncol. 117: 83-90.

17) Marks L et al. ( 2010) Use of Normal Tissue Complication Probability Models in the Clinic. International Journal of Radiation Oncology ${ }^{\star}$ Biology ${ }^{\star}$ Physics. 76: S10-S19.

18) (2009) National Cancer Institute. Common Terminology Criteria for Adverse Events (CTCAE) Version 4.0. Published: May 28, (v4.03: June 14, 2010) .
19) Huang C J et al. (2015) RTOG, CTCAE and WHO criteria for acute radiation dermatites correlate with cutaneous blood flow measurements. Breast. $24: 230$

20) Paula J, Sawada N O (2015) Qualidade de vida relacionada à saúde de pacientes com câncer em tratamento radioterápico. Rev Rene, 16 : 106-113,.

21) GALBIATTI A et al. (2012) Head and neck cancer: genetic polymorphisms and folate metabolism. Braz J Otorhinolaryngol, 78 : 132$139 . S$

22) Poon C, Kerstin M Overview of the diagnosis and staging of head and neck cancer. UpToDate2015.

23) AMERICAN CANCER SOCIETY. Cancer facts \& figures 2018. Atlanta, 2018.

24) CHUFAL K. et al. (2006) Analysis of prognostic variables among patients with locally advanced head and neck cancer treated with late chemo-intensification protocol: impact of nodal density and total tumor volume. Jpn J ClinOncol, 36: 537-46.

25) Poulsen $\mathrm{M}$ et al. (2008) Predictors of acute grade 4 swallowing toxicity in patients with stages III and IV squamous carcinoma of the head and neck treated with radiotherapy alone. RadiotherOncol, 87: 253-259.

26) Alencar A (2011) Tratamento da mucosite oral induzida por radio e quimioinduzida: comparação entre protocolo medicamentoso convencional e tratamentos com lasers em baixa intensidade. Dissertação. São Paulo: Universidade de São Paulo; Instituto de Pesquisas Energéticas e Nucleare.

27) Matuschek $C$ et al. (2016) Influence of dosimetric and clinical criteria on the requirement of artificial nutrition during radiotherapy of head and neck cancer patients. RadiotherOncol 120: 28-35.

28) Lionel D, Christophe L, Marc A, Jean-Luc C (2006) Oral mucositis induced by anticancer treatments: physiopathology and treatments. Ther Clin Risk Manag 2: 159-168.

29) 29. Rodriguez-Caballero A et al. (2012) Cancer treatment-induced oral mucositis: a critical review. Int J Oral MaxillofacSurg 41: 225-238.

30) Hsu P-Y et al. (2016) Efficacy of Traditional Chinese Medicine in Xerostomia and Quality of Life during Radiotherapy for Head and Neck Cancer: A Prospective Pilot Study. Evidence-based Complementary and Alternative Medicine : eCAM, 2016: 8359251.

31) Fávaro R,Ferreira T, Martins W (2006) Xerostomia: etiologia, diagnóstico e tratamento. Clin. Pesq. Odontol, Curitiba 2 : 303-317.

\section{Submit your manuscript to a JScholar journal} and benefit from:

ब Convenient online submission

I Rigorous peer review

ฯ Immediate publication on acceptance

बा Open access: articles freely available online

ब High visibility within the field

- Better discount for your subsequent articles Submit your manuscript at http://www.jscholaronline.org/submit-manuscript.php 